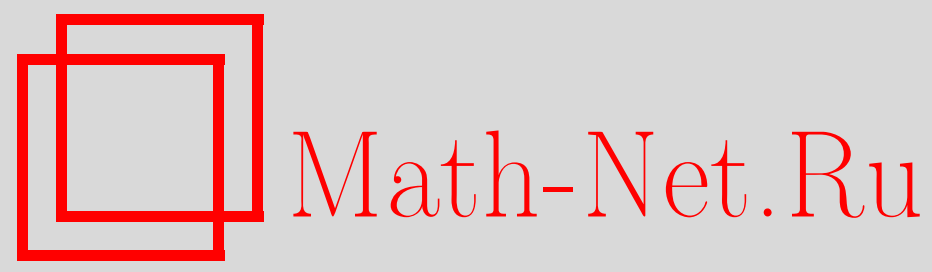

А. А. Махнев, Аффинные овоиды и расширения обобщенных четырехугольников, Матем. заметки, 2000, том 68, выпуск 2, 266-271

DOI: https://doi.org/10.4213/mzm943

Использование Общероссийского математического портала Math-Net.Ru подразумевает, что вы прочитали и согласны с пользовательским соглашением http://www.mathnet.ru/rus/agreement

Параметры загрузки:

IP: 54.162 .85 .209

26 апреля 2023 г., 03:50:35

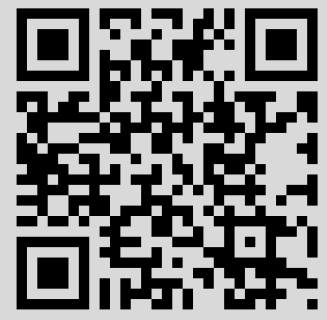




\section{АФФИННЫЕ ОВОИДЫ И РАСШИРЕНИЯ ОБОБЩЕННЫХ ЧЕТЫРЕХУГОЛЬНИКОВ}

\section{А. А. Махнев}

Подмножество вершин $\Delta$ обобщенного четырехугольника порядка $(s, t)$ называется гиперовалом, если каждая прямая пересекает $\Delta$ по 0 или 2 точкам. Гиперовал $\Delta$ называется аффинным овоидом, если $|\Delta|=2 s t$. Хорошо известно, что $\mu$-подграфы в треугольных расширениях обобщенных четырехугольников являются гиперовалами. Доказано, что если $\mathscr{S}$ является треугольным расширением для $G Q(s, t)$ с вполне регулярньм точечным графом $\Gamma$, имеющим $\mu=2 s t$, то $s$ четно, $\Gamma$ является $r$-антиподальным графом диаметра 3 с $r=1+s / 2$ и либо $s=2$, либо $t=s+2$.

Библиография: 8 названий.

Мы рассматриваем неориентированные графы без петель и кратных ребер. Пусть $X$ - некоторое множество вершин графа $\Gamma$. В данной работе подграф $X$ будет обозначать подграф, индуцированньй $\Gamma$ на $X$. Если $a, b$ вершины графа $\Gamma$, то через $d(a, b)$ обозначим расстояние между $a$ и $b$, а через $\Gamma_{i}(a)$ - подграф на множестве всех вершин графа $\Gamma$, которые находятся на расстоянии $i$ от вершины $a$. Подграф $\Gamma_{1}(a)$ мы будем назьвать окрестностью вершины $a$ и обозначать через $[a]$. Через $a^{\perp}$ обозначим подграф $\{a\} \cup[a]$.

Валентностью вершины назьвается число вершин в ее окрестности. Граф Г назовем регулярным валентности $k$, если валентность любой вершины $a$ из Г равна $k$. Граф Г назовем реберно регулярным с параметрами $(v, k, \lambda)$, если он содержит $v$ вершин, регулярен валентности $k$ и каждое его ребро лежит в $\lambda$ треугольниках. Граф $\Gamma$ вполне регулярен с параметрами $(v, k, \lambda, \mu)$, если он реберно регулярен с соответствуюшими параметрами и $[a] \cap[b]$ содержит $\mu$ вершин для любых двух вершин $a, b$, находящихся на расстоянии 2 в Г. Вполне регулярный граф диаметра 2 назьвается сильно регулярным. Полньй многодольный граф с $n$ долями порядка $m$ назовем $K_{n \times m}$-графом. Подграф $[a] \cap[b]$ назовем $(\lambda-) \mu$-подграфом, если вершины $a, b$ (смежны) находятся на расстоянии 2 .

Геометрией ранга 2 назьвается система инцидентности $\mathscr{S}=(P, B)$ с множеством точек $P$ и семейством подмножеств $B$ из $P$ (называемых блоками). Две точки из $P$ назьваются коллинеарнылми, если они лежат в общем блоке (заметим, что точка коллинеарна себе). Коллинеарность точек $x$ и $y$ (двойственно, факт пересечения блоков $L$ и $M$ ) мы будем обозначать как $x \sim y$ (соответственно $L \sim M$ ). Для точки $x$ положим $x^{\perp}=\{y \in P \mid y \sim x\}$. Вычетом геометрии $\mathscr{S}$ в точке $x$ называется геометрия

Работа выполнена при финансовой поддержке Российского фонда фундаментальных исследований, грант № 99-01-00462. 
$\mathscr{S}_{x}=\left(P_{x}, B_{x}\right)$, где $P_{x}=x^{\perp}-\{x\}, B_{x}=\{L-\{x\} \mid x \in L, L \in B\}$. Геометрия называется треугольной, если любые ее три попарно коллинеарные точки лежат в общем блоке. Пусть $\mathscr{F}$ - класс геометрий ранга 2. Геометрия $\mathscr{S}$ назьвается $\mathscr{F}$-расширением, если вычет $\mathscr{F}$ в каждой точке принадлежит $\mathscr{F}$. Если $a \in P, L \in B$ и $a \notin L$, то пара $(a, L)$ называется антифлагом. Число точек из $L$, коллинеарных с $a$, обозначается через $f(a, L)$. Геометрия $G$ называется $\varphi$-однородной, если $f(a, L)=0$ или $f(a, L)=\varphi$ для любого антифлага $(a, L) ; G$ назьвается сильно $\varphi$-однородной, если $f(a, L)=\varphi$ для любого антифлага $(a, L)$. Если в некоторой геометрии любые два различных блока пересекаются не более чем по одной точке, то множество блоков будем называть множеством прямых.

Геометрия, состоящая из точек и прямых, называется обобщенным четырехугольником порядка $(s, t)$, если каждая прямая содержит $s+1$ точку, каждая точка лежит на $t+1$ прямой (две прямые пересекаются не более чем по одной точке) и для любой точки $a$, не лежащей на прямой $L$, найдется единственная прямая, проходящая через $a$ и пересекаюшая $L$ (геометрия сильно 1-однородна). Через $G Q(s, t)$ будем обозначать класс всех обобщенных четырехугольников порядка $(s, t)$.

Пусть $\mathscr{S}$ является обобщенньп четырехугольником порядка $(s, t)$. Точечным графом $\mathscr{S}$ называется граф, вершинами которого являются точки $\mathscr{S}$, и две различные вершины смежны, если они лежат на общей прямой. Легко понять, что точечный граф $\Gamma$ для $\mathscr{S}$ сильно регулярен с параметрами: $v=(s+1)(s t+1), k=s(t+1), \lambda=s-1$, $\mu=t+1$. Подграф $\Delta$ из $\Gamma$ называется гиперовалом, если каждая прямая пересекает $\Delta$ по 0 или 2 точкам (при этом прямая назьвается внешней или секущей соответственно). Хорошо известно [1], что $\min \{2(t+1),(s+1)(t+2-s)\} \leqslant|\Delta| \leqslant 2(s t+1)$. Если $|\Delta|=2(t+1)$, то $\Delta$ является двойственной решеткой $K_{2 \times(t+1)}$, если же $|\Delta|=2(s t+1)$, то каждая прямая $\mathscr{S}$ пересекает $\Delta$ по двум точкам и $\Delta$ называется двойным овоидом. Гиперовал $\Delta$ называется аффиннылм овоидом, если $|\Delta|=2 s t$. Для любого аффинного овоида $\Delta$ найдется точка $a$ такая, что множество внешних прямых совпадает с множеством прямых $\mathscr{S}$, проходящих через $a$. Точка $a$ называется бесконечно удаленной от $\Delta$. Таким образом, $\Delta$ является двойным овоидом "аффинного обобщенного четырехугольника" $\mathscr{S}-a^{\perp}$.

Изучение треугольных (2-однородных) $G Q(s, t)$-расширений эквивалентно изучению локально $G Q(s, t)$-графов, т.е. графов, в которых окрестности вершин изоморфны точечным графам обобщенных четырехугольников порядка $(s, t)$. Обзор результатов по расширениям обобщенных четырехугольников можно найти в [1]. Особенно просто устроены $G Q(1, t)$-расширения. Это геометрии вершин и 3 -клик графов $K_{3 \times(t+1)}$. Пусть $\mathscr{S}$ является треугольньм $G Q(s, t)$-расширением с точечным графом $\Gamma$. Тогда любой $\mu$-подг раф $\Delta=[a] \cap[b]$ является гиперовалом в обобщенных четырехугольниках $[a]$ и $[b]$. Экстремальные случаи $|\Delta|=2(t+1)$ и $|\Delta|=2(s t+1)$ рассматривались в работах [2], [3]. При этом в [3] классифицированы все флаг-транзитивные расширения обобшенных четырехугольников. Эти расширения (в случае $s>2$ ) приведены в примерах 1,2 .

ПримеР 1. Пусть геометрия $\mathscr{G}$ - флаг-транзитивное $G Q(s, t)$-расширение с $s>2$ и $\mu$-подграфами, являюшимися двойственными решетками $K_{2 \times(t+1)}$. Тогда возможны только следующие случаи:

1) $\mathscr{G}$ является локально $W(3)$ с группой автоморфизмов $U_{5}(2) .2$;

2) $\mathscr{G}$ является локально $H_{3}\left(p^{2}\right), p=2$ или 3 , с группой автоморфизмов 3.( $\left.O_{6}^{-}(3) .2\right)$ или $S u z .2$ соответственно. 
ПримеР 2. Пусть геометрия $\mathscr{G}$ - флаг-транзитивное $G Q(s, t)$-расширение с $s>2$ и $\mu$-подграфами, являющимися двойными овоидами. Тогда возможны только следующие случаи:

1) $\mathscr{G}$ является локально $Q_{5}^{-}(3)$ с группой автоморфизмов $M c L .2$;

2) $\mathscr{G}$ является локально $H_{3}\left(p^{2}\right), p=2$, с группой автоморфизмов $O_{6}^{-}(3) .2$.

В нашей работе исследуется один из двух, ближайших к рассмотренньм случаев, а именно, $|\Delta|=2 s t$ для любого $\mu$-подграфа $\Delta$. В этом случае $\mu$-подграфы из Г являются аффинными овоидами. Граф Г диаметра $d$ называется $r$-антиподальным, если отношение “совпадать или находиться на расстоянии $d$ ” является отношением эквивалентности на множестве вершин графа и $r$ - число вершин в каждом классе эквивалентности. Если $\bar{\Gamma}$ - факторграф по этому отношению эквивалентности, то Г назьвается $r$-накрытием $\bar{\Gamma}$. В случае $r=2$ и $d=3$ накрытие клики назьвается графом Тэйлора.

ТЕОремА. Пусть $\mathscr{G}$ является треугольным $G Q(s, t)$-расширением, $s>1$, с вполне регулярным точечным графом $Г$, имеющим $\mu=2 s t$. Тогда $s$ четно, Г является г-антиподальным графом диаметра 3 с $r=1+s / 2$ и выполняется одно из утверәдений:

1) $s=2 u$ Гявляется графом Тәйлора;

2) $t=s+2$.

Эта теорема является усилением одного результата Пазини [4, следствие 3], в котором дополнительно предполагалось, что все вычеты являются классическими обобщенными четырехугольниками, а в заключении утверждалось лишь, что Г является $r$-антиподальным графом диаметра $3 \mathrm{c} r=1+s / 2$. Фактически, в случае 1$)$ для каждого $t=1,2$ или 4 существует единственный локально $G Q(2, t)$ граф Тэйлора, а в случае 2) для каждого $q=p^{m}, p$ нечетное простое, существует треугольное $G Q(q-1, q+1)$-расширение с $\mu=2 q^{2}-2$, вычетами которого являются обобщенные четырехугольники Аренса-Секереша $A S(q)$. Напомним, что классическим обобщенным четырехугольником назьвается обобщенный четырехугольник, точками и прямыми которого являются

1) сингулярные точки и вполне сингулярные прямые несингулярной квадрики $Q$ из $P G(d, q)$ с индексом Витта, равньм $1, d=3,4$ или 5 ;

2) сингулярные точки и вполне сингулярные прямые несингулярной эрмитовой формы $H$ на $P G\left(d, q^{2}\right), d=3$ или 4 ;

3) изотропные точки и вполне изотропные прямые невырожденной знакопеременной формы $W$ на $P G(3, q)$.

Приступим к доказательству теоремы. Сначала приведем один вспомогательньй результат.

ЛЕмма 1. Пусть вполне регулярный граф Г с параметрами $(v, k, \lambda, \mu)$ является r-накрытием п-клики. Тогда выполняются следующие утверждения:

1) Г дистаниионно регулярен, $n=k+1$ и имеет место равенство $n-2-\lambda=$ $(r-1) \mu$

2) матрица смежсности Г имеет собственные значения $n-1,1, \theta, \tau$ кратностей $1, n-1, m_{\theta}, m_{\tau}$, әде $\tau+\theta=\lambda-\mu, \theta \tau=1-n$,

$$
m_{\theta}=\frac{n(r-1) \tau}{\tau-\theta}, \quad m_{\tau}=\frac{n(r-1) \theta}{\theta-\tau}
$$


3) выполняются следующие соотношения мехду параметрами накрытия:

a) $1 \leqslant(r-1) \mu \leqslant n-2$

b) если п четно, то и н четно;

c) $\theta, \tau$ - иелье числа разных знаков, поэтому можно считать, что $\theta>0$;

d) если $r>2$, mo $\theta^{3} \geqslant n-1$.

ДокАЗАТЕЛЬСТво. См. статью [5].

Пусть обобщенный четырехугольник $\mathscr{S}$ порядка $(s, t)$ содержит аффинньй овоид $\Delta$. Множество секущих $\Delta$ (внешних прямых) обозначим через $L_{\Delta}^{+}$(через $L_{\Delta}^{-}$).

Лемма 2. Найдется точка $a=a_{\Delta}$ такая, что $L_{\Delta}^{-}$совпадает с мнохсеством прямых $\mathscr{S}$, проходящ, их через а.

ДоКАЗАТЕЛЬСтво. Ясно, что $\left|L_{\Delta}^{+}\right|=s t(t+1)$ и $\left|L_{\Delta}^{-}\right|=t+1$. Выберем прямую $L$ из $L_{\Delta}^{-}$. Каждая прямая из $L_{\Delta}^{+}$, пересекающая $L$, содержит две точки из $\Delta$, и каждая точка из $\Delta$ лежит на одной из таких прямых. Отсюда ровно $s t$ прямых из $L_{\Delta}^{+}$пересекают $L$, и оставшиеся $t+1$ прямых, пересекающих $L$, принадлежат $L_{\Delta}^{-}$. Таким образом, любые две прямые из $L_{\Delta}^{-}$пересекаются, следовательно, $L_{\Delta}^{-}$совпадает с множеством прямых $\mathscr{S}$, проходящих через некоторую точку $a$. Лемма доказана.

Пусть до конща работы $\mathscr{G}$ является треугольным $G Q(s, t)$-расширением с вполне регулярным точечным графом $\Gamma$, имеющим $\mu=2 s t$. Следуя Пазини [4], для точек $a, b \in \mathscr{G}$, находящихся на расстоянии 2 в $\Gamma$, через $b(a)$ обозначим точку из $[a]$, бесконечно удаленную от $[a] \cap[b]$.

Лемма 3. Выполняются следующие утверждения:

1) точки $b, b(a)$ находятся на расстоянии 3 в Г;

2) Г является $r$-антиподальным графом диаметра 3 с r $=1+s / 2$.

ДоКАЗАТЕЛЬСтво. Допустим, что точки $b, b(a)$ находятся на расстоянии 2 в $Г$. Тогда диаметр графа Г равен 2 и Г сильно регулярен с параметрами $k=(s+1)(s t+1)$, $\lambda=s(t+1), \mu=2 s t$. Так как $\lambda \neq \mu-1$, то $(\lambda-\mu)^{2}+4(k-\mu)=n^{2}$ для некоторого натурального $n$. Теперь $n^{2}=s^{2}(t+1)^{2}-4 s t+4 s+4$ и $(s t+s-1)^{2}=(s t+s)^{2}-2 s t-2 s+1$, поэтому $-4 s t+4 s+4=-2 s t-2 s+1$, противоречие. Утверждение 1) доказано.

Заметим, что $c=b(a)$ - единственная точка из $[a]$, находящаяся на расстоянии 3 от $b$. Если $d \in[c] \cap \Gamma_{2}(b)$, то $c=b(d)$ и все точки из $[d]-\{c\}$ находятся на расстоянии не большем 2 от $b$. Так как $\left|\Gamma_{2}(b)\right|=s(s+1)(s t+1) / 2$, то имеется точно $s / 2$ точек, находящихся на расстоянии 3 от $b$, причем $[c] \cap\left[c^{\prime}\right]$ не пересекает $\Gamma_{2}(b)$ для любых различных точек $c, c^{\prime} \in \Gamma_{3}(b)$. Таким образом, $\Gamma$ является $r$-антиподальным графом диаметра $3 \mathrm{c}$ $r=1+s / 2$. Лемма доказана.

Теперь по лемме 1 граф $Г$ имеет собственные значения $\theta=s+1, \tau=-(s t+1) \mathrm{c}$ кратностями

$$
m_{\theta}=\frac{(k+1) s(s t+1)}{2(s t+s+2)}, \quad m_{\tau}=\frac{(k+1) s(s+1)}{2(s t+s+2)} .
$$

ЛЕмма 4. Если $s=2$, то Г- граф Тәйлора.

ДокАЗАТЕЛЬСтво. Это утверждение следует из леммы 3.

В [1] отмечено, что для каждого $t=1,2,4$ существует единственный локально $G Q(2$, t)-граф Тэйлора. 
ЛЕмма 5. Ecлu $t=1$, mo $s=2$.

ДокАЗАТЕльство. Предположим, что $t=1$ и $s \geqslant 4$. Тогда Г является локально $(s+1) \times(s+1)$-решетчатым граффом. Назовем блоками $(s+2)$-клики из Г. Положим $m=s / 2$. Тогда выполняются следующие утверждения:

1) если $C$ - блок, $x \notin C$, то точка $x$ смежна с 0 или 2 точками блока $C$;

2) любое ребро из $Г$ лежит точно в двух блоках, а любой треугольник из Г лежит в единственном блоке;

$3)$ если $a \in \Gamma,\left\{a_{1}^{*}, \ldots, a_{m}^{*}\right\}=\Gamma_{3}(a)$, то для любой вершины $b=b_{1} \in\left[a_{1}^{*}\right]$ найдутся $b_{i} \in\left[a_{i}^{*}\right], i=2, \ldots, m$, такие, что $\left\{b(a), b_{2}, \ldots, b_{m}\right\}=\Gamma_{3}(b)$, при этом $(s \times s)$-решетка $[a]-b(a)^{\perp}$ расшепляется $m$ подграфами $\Delta_{i}=[a] \cap\left[b_{i}\right]$, каждьй из которых является объединением изолированных многоугольников с четным числом вершин.

Утверждения 1) и 2) очевидны. Подграф $\Delta_{i}$ имеет валентность 2 , поэтому он является объединением изолированных многоугольников. Если некоторое ребро многоугольника лежит на горизонтальной прямой решетки $[a]$, то инцидентные с ним ребра этого многоугольника лежат на вертикальных прямых $[a]$. Это доказьвает последнее утверждение из 3).

Для точки $x \in[b] \cap \Gamma_{2}(a)$ пересечение $[x] \cap \Delta_{1}$ является ребром, если $x \in[a(b)]$; состоит из двух неинцидентных ребер, одно из которых лежит на вертикальной прямой из $[a]$, а другое - на горизонтальной, если $x \notin[a(b)]$.

Пусть $L$ - горизонтальная прямая из $[a]$, проходящая через $b(a), L_{i}^{*}-$ прямая из $\left[a_{i}^{*}\right]$, состояшая из антиподов для точек прямой $L$. Для $x \in L_{1}^{*}-\{b\}$ подграф $[a] \cap[x]$ содержит ребро с вертикальной прямой $M$ из $[a]$, проходящей через точку $b(a)$, ребро с горизонтальной прямой $[a]$, пересекающей $\Delta_{1}$, а также $2 s-4$ точки из $\cup\left\{\Delta_{i} \mid i=2, \ldots, m\right\}$.

Число ребер в $M-\{b(a)\}$ равно $s(s-1) / 2$. Пусть $b_{i}$ - антипод точки $b(a)$, принадлежащий $L_{i}^{*}$. Тогда окрестность каждой точки из $L_{i}^{*}-\left\{b_{i}\right\}$ содержит ребро из $M-\{b(a)\}$, причем $\left|\cup\left\{L_{i}^{*}-\left\{b_{i}\right\} \mid i=1, \ldots, m\right\}\right|=s^{2} / 2$. Значит, некоторое ребро из $M-\{b(a)\}$ попадает в $[x] \cap[y]$ для $x \in L_{i}^{*}, y \in L_{j}^{*}, i \neq j$. По утверждению 2) вершины $x, y$ смежны. Противоречие с тем, что прямая $L_{i}^{*}$ содержит точку, антиподальную к $y$.

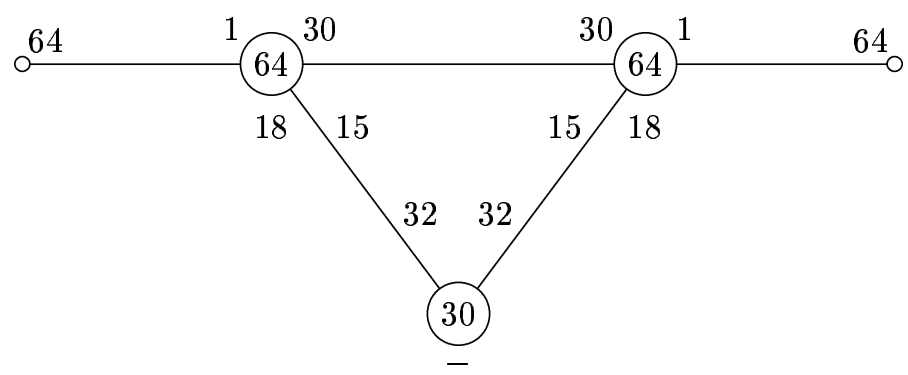

Рис. 1. Локально $G Q(3,5)$-граф

ЛЕмма 6. Если $t>1 u s>2, \operatorname{mot}=s+2$.

ДокАЗАТЕЛЬСтво. Если $s>2$, то $r>2$, и по утверждению d) леммы $1(s+1)^{3} \geqslant$ $(s+1)(s t+1)$, т.е. $s \geqslant t-2$.

По условию целочисленности $2(s t+s+2)$ делит $\left(s^{2} t+s t+s+2\right) s(s+1)$. Далее, $(s t+s+2, s+1)=(t-1, s+1),(s t+s+2, k+1)=\left(s t+s+2, s^{2} t\right)=(s t+s+2, s(s+2))$. 
Наконец, $(s t+s+2, s+2)=(2 t, s+2)$ и $s t+s+2$ делит $2(s+2)(t-1)$. Поэтому $s t+s+2$ делит $4(s+2-t)$. Предположим, что $t \neq s+2$. Тогда $t<4$. Если $t=2$, то $s=2$. Если же $t=3$, то $4 s+2$ делит $2(s-1)$, противоречие. Лемма, а вместе с ней и теорема доказаны.

Существование треугольных $G Q(q-1, q+1)$-расширений, вычетами которых являются обобщенные четырехугольники Аренса-Секереша $A S(q)$, отмечено в примере 9.15 c $m=(q+2) / 2[1]$.

В связи с доказанной теоремой вызывает интерес следующая

ПроБЛЕмА. Описать класс геометрий $\mathscr{F}$, состоящий из треугольных $G Q(s, t)$ расширений с точечным графом Г диаметра большего 2 таких, что н-подграфы вершин, лежсаших в геодезических 3-путях графа Г, являются аффинными овоидамu.

Следующие геометрии удовлетворяют всем условиям, перечисленньм в этой проблеме.

ПРИмеР 3. Связньй локально $G Q(3,5)$-граф имеет диаграмму, изображенную на рис. 1 , относительно любой вершины (Махнев [6]). Существование геометрии $\mathscr{G}$, являющейся флаг-транзитивным треугольным $G Q(3,5)$-расширением, установлено Ешиарой [7].

ПримеР 4. Геометрия из примера 3 является наименьшей в бесконечной серии $\widetilde{\Gamma}_{q}$ треугольных $G Q(q-1, q+1)$-расширений, $q=2^{n}, n \geqslant 2$. Точечньй граф $\widetilde{\Gamma}_{q}$ является $q / 2$-накрытием полного многодольного графа $K_{(q+1) \times q^{2}}$ (Дель $\Phi$ ра, Пасечник, Пазини [8]).

\section{СПИСОК ЦИТИРОВАННОЙ ЛИТЕРАТУРЫ}

[1] Cameron P., Hughes D.R., Pasini A. Extended generalized quadrangles // Geom. Dedicata. 1990. V. 35. P. 193-228.

[2] Fisher P., Hobart S. Triangular extended generalized quadrangles // Geom. Dedicata. 1991. V. 37. P. 339-344.

[3] Del Fra A., Ghinelli D., Meixner T., Pasini A. Flag-transitive extensions of $C_{n}$ geometries // Geom. Dedicata. 1992. V. 37. P. 253-273.

[4] Pasini A. Remarks on double ovoids in finite classical generalized quadrangles, with an application to extended generalized quadrangles // Quart. J. Pure Appl. Math. 1992. V. 66. P. 41-68.

[5] Godsil C. D. Covers of complete graphs // Adv. Stud. Pure Math. 1996. V. 24. P. 137-163.

[6] Махнев А. А. Локально $G Q(3,5)$-грабфы и геометрии с короткими прямыми // Дискретная матем. 1998. Т. 10. С. 72-86.

[7] Yoshiara S. On some flag-transitive non-classical c.C $C_{2}$-geometries // Europ. J. Combin. 1993. V. 14. P. 59-77.

[8] Del Fra A., Pasechnik D. V., Pasini A. A new family of extended generalized quadrangles // Europ. J. Combin. 1997. V. 18. P. 155-169. 